\title{
FLUID FLOW IN A LAYERED MEDIUM
}

\author{
BY
}

\author{
G. W. CLARK AND R. E. SHOWALTER
}

The University of Texas at Austin, Austin, Texas

\begin{abstract}
A layered medium is modeled as a continuous distribution of relatively flat cells within a spatial region. Local flow within each cell as well as the exchange with the global flow over the region is modeled by a quasilinear parabolic system of partial differential equations, and the local geometry of the individual cells is included in the model. We introduce new terms to account for the secondary flux corresponding to either transverse flow across the cells or direct cell-to-cell diffusion driven by the global density gradient. The resulting initial-boundary-value problem is shown to be well-posed and to depend continuously on the parameter defining the type of interface condition on cell boundaries.
\end{abstract}

1. Introduction. We will begin by presenting a few models of fluid flow through a fractured reservoir. Barenblatt et al. [5] describe flow through a general heterogeneous medium by modeling it as two overlapping media. Two fluid pressures are introduced, each representing the fluid pressure in the corresponding medium. The resulting system of coupled equations takes the form

$$
\begin{aligned}
\theta_{11} \partial_{t} v-\theta_{12} \partial_{t} w+\alpha(v-w) & =\vec{\nabla} \cdot\left(D_{1} \vec{\nabla} v\right), \\
-\theta_{21} \partial_{t} v+\theta_{22} \partial_{t} w-\alpha(v-w) & =\vec{\nabla} \cdot\left(D_{2} \vec{\nabla} w\right),
\end{aligned}
$$

where $v$ represents the fluid density in the first medium and $w$ represents the density in the second. Here the $\theta_{i j}$ 's represent the saturation constants or porosities of the two media and $D_{1}$ and $D_{2}$ give the permeability of the media. This type of model is generally referred to as a parallel flow model. Several variations of the above can be obtained by adjusting the coefficients. For example, if one specializes this to a matrix of permeable blocks separated from each other by a system of fissures and assumes that the blocks are completely isolated from each other by the fissure system, we set $D_{2}=0$. The resulting first-order kinetic system models fluid flow in which the individual blocks interact only with the surrounding fissure system.

\section{Received August 18, 1992.}

1991 Mathematics Subject Classification. Primary 35K55, 35K65.

Key words and phrases. Porous medium, double porosity, partially fissured medium, microstructure, quasilinear parabolic system, existence, uniqueness.

This work was supported by grants from the National Science Foundation and the Office of Naval Research.

(c) 1994 Brown University 
For a layered medium one needs anisotropic assumptions. In a simple model consisting of horizontal alternating layers of block and fissure, Boulton and StreltsovaAdams [6] assume that the flow in the fissures is horizontal while fluid flows only vertically in the blocks. In (1.1) we might represent such a situation by defining the terms $D_{1}$ and $D_{2}$ to be $3 \times 3$ matrices. The flow assumptions could then be modeled by adjusting the coefficients of the corresponding matrix. Kasap and Lake [9] approach a similar problem involving flow in a layered medium by calculating an effective permeability of the block-fissure system. In this approach, a heterogeneous block is replaced by a homogeneous one. The permeability of the homogeneous block corresponds to the effective permeability of the heterogeneous one so that the critical aspects of the fluid flow are preserved.

In the Barenblatt model described above, the exchange terms are assumed to be proportional to the difference in fluid density between block and fissure. We will present a model in which the geometry of the blocks plays a role in this flux exchange. To accomplish this we use the concept of the distributed microstructure model. These models utilize two scales to describe the medium of blocks and fissures. At each point in the fissure, or macro-scale, is specified a block, or cell, which exists in the micro-scale. The fissures affect the cells on their boundaries in the micro-scale while the cell effects are spatially distributed throughout the fissure system. Such models have been used recently by Arbogast [2,3] and Arbogast, Douglas, and Hornung [4] in oil reservoir simulation, by Hornung and Showalter [8], and by Showalter and Walkington [16, 17], and they also appeared in numerous earlier works.

We will introduce and develop a double porosity microstructure model for a medium with a layered structure. Our assumptions are similar to those of Boulton and Streltsova-Adams described above. When dealing with a horizontally layered matrix of blocks and fissures we will consider only vertical flow within the blocks or cells. In previous models these cells have been viewed as storage locations only. Fluid passes from the fissure system into the cells, is stored there for a time, and then exits the cell back into the fissure system. A significant difference between such models and those introduced here is that, in addition to this storage effect, the fluid here is forced through the cell system by pressure differences in the fissure system. This flow in the cells produces an additional component to the velocity field in the fissure system which we will refer to as the secondary flux.

In the following section we will describe the physical setting for the microstructure models. We will compute an expression for the exchange of fluid between the individual cells and the fissure system which accounts for the secondary effects of both storage and flux. This will allow us to write a system of partial differential equations, coupled by this exchange term and by boundary values, which effectively models the flow of fluid in the medium. We can also write the system as a single functional PDE. The latter sections of this work contain the mathematical formulation of our microstructure models. In Sec. 3 we pose these problems with the new secondary flux effects in a variational setting on spaces constructed using continuous direct sums of various Banach spaces of Sobolev type. Finally, in Sec. 4 we prove a general theorem on the existence and uniqueness of solutions of the variational equations which 
represent the models and give a result on the continuous dependence of solutions on the type of boundary condition.

2. The model. The medium modeled here consists of a matrix of fissures and cells in which the individual cells interact only with the surrounding fissure system. We are interested in a layered structure, which is modeled by a continuous distribution of thin cells throughout the region. The fissure system might occupy little volume relative to the total volume of the cells, but it has a much higher permeability. Although most of the transport of fluid occurs in the fissure system, much of the fluid may be located within the cells at any given time. Because of the shape of the cells and the higher permeability of the fissure material, a pressure difference in the vicinity of a cell tangential to the associated foliation would permit fluid to pass easily around the cell but induce little or no flow within the cell. However, the same pressure difference in the normal direction induces fluid to flow through the cell. In the following we will assume that flow within the cell responds to a fissure pressure gradient perpendicular to the layer in a neighborhood of the cell.

We require some notation in order to proceed; so let $\Omega \subset \mathbb{R}^{3}$ be a bounded open set and at each point $x \in \Omega$, let $\Omega_{x}$ represent the cell structure within a neighborhood of that point. Let $u(x, t)$ and $U(x, y, t)$ represent the fluid density in the fissures and cells respectively for $x \in \Omega, y \in \Omega_{x}, t>0$. It should be emphasized that two different scales are implicitly employed here. In the macro-scale, any $x \in \Omega$ is a point in the fissure system and $u(x, t)$ represents the value of the fluid density at that point. In the micro-scale, every $\Omega_{x}$ represents an individual cell with the $y$ variable determining the location within the cell. Thus $U(x, y, t)$ gives the fluid density in the cell $\Omega_{x}$ at the point $y$.

Flow in the global fissure system is assumed to be governed by the classical diffusion equation

$$
\frac{\partial}{\partial t}(a(x) u(x, t))-\vec{\nabla} \cdot(A(x) \vec{\nabla} u(x, t))+Q(x, t)=f(x, t), \quad x \in \Omega,
$$

where $f(x, t)$ is a prescribed distributed source and $Q(x, t)$ represents the density of fluid flow from the fissure system into the cell $\Omega_{x}$ across its boundary $\Gamma_{x}$. The effect of the cells on the fissure system is distributed throughout the region $\Omega$ with each cell acting as a source or sink, while the fissure system affects the cells through their boundaries.

The cells $\Omega_{x}$ used to model this layered media have dimensions which are much smaller in one direction than in the other two directions, so that they are nearly flat. Let $T_{x}$ be a (two-dimensional) plane passing through $\Omega_{x}$ and perpendicular to the direction given by the (small) height of $\Omega_{x}$. Choose a coordinate system so that for $y \in \Omega_{x}$,

$$
y=\left(y_{1}, y_{2}, y_{3}\right)=\left(y_{1}, y_{2}, 0\right)+y_{3} \vec{n}_{x}
$$

where $\left(y_{1}, y_{2}, 0\right) \in T_{x} \cap \Omega_{x}$ and $\vec{n}_{x}$ is a unit vector normal to $T_{x}$. Note that $\vec{n}_{x}$ gives the direction of the secondary flux. In each cell we assume that the flow is governed by the diffusion equation

$$
\frac{\partial}{\partial t}(b(x, y) U(x, y, t))-\vec{\nabla}_{y} \cdot\left(B(x, y) \vec{\nabla}_{y} U(x, y, t)\right)=0
$$


along with a constraint on the cell boundary $\Gamma_{x} \equiv \partial \Omega_{x}$ of the form

$$
U(x, s, t)=g(x, s, t), \quad s \in \Gamma_{x},
$$

where $g$ represents the influence of the surrounding fissures on the cell. In all of the previous work involving this type of model, only the effect of the fissure fluid density in a neighborhood of the cell has been considered, i.e., $g(x, s, t)=u(x, t)$, $s \in \Gamma_{x}$. We shall incorporate into this model both the effect of the local value of the fluid density on the cell and the effect of the density gradient across the cell. We will ignore the effect of the gradient of density in the $y_{1}$ and $y_{2}$ directions, because the effective cell permeability is much lower than that of the fissures. Thus we approximate $u(x+\vec{\xi})$ for $x+\vec{\xi} \in \Gamma_{x}$ by

$$
u(x+\vec{\xi}) \cong u(x)+\left(\vec{\xi} \cdot \vec{n}_{x}\right) \vec{\nabla} u(x) \cdot \vec{n}_{x} .
$$

In the cell problem above we define the function $g$ representing this influence by

$$
g(x, s, t)=u(x, t)+s_{3} \vec{\nabla} u(x, t) \cdot \vec{n}_{x} .
$$

The coupled system representing the fissures and cells can now be written

$$
\begin{aligned}
& \frac{\partial}{\partial t}(a(x) u(x, t))-\vec{\nabla} \cdot(A(x) \vec{\nabla} u(x, t))+Q(x, t)=f(x, t), \quad x \in \Omega, t>0 ; \\
& \begin{cases}\frac{\partial}{\partial t}(b(x, y) U(x, y, t))-\vec{\nabla}_{y} \cdot\left(B(x, y) \vec{\nabla}_{y} U(x, y, t)\right)=0, & y \in \Omega_{x} ; \\
U(x, s, t)=u(x, t)+s_{3} \vec{\nabla} u(x, t) \cdot \vec{n}_{x}, & s \in \partial \Omega_{x} \equiv \Gamma_{x} .\end{cases}
\end{aligned}
$$

We shall also assume that $u$ satisfies the Dirichlet boundary condition

$$
u(x, t)=0, \quad x \in \partial \Omega,
$$

although everything can be achieved likewise for the other types of boundary conditions.

To determine $Q(x, t)$ we will consider the normal first-order effects of the cells on the fissure system. The rate at which fluid flows across $\Gamma_{x}$ is given by $B(x, s) \frac{\partial U}{\partial \nu}(x, s, t), s \in \Gamma_{x}, x \in \Omega$. We compute its effect as a distribution on test functions of the form

$$
\Phi(x, y)=\varphi(x)+y_{3} \vec{\nabla} \varphi(x) \cdot \vec{n}_{x}, \quad \varphi \in C_{0}^{\infty}(\Omega),
$$

as follows:

$$
\begin{aligned}
\int_{\Omega} \int_{\Gamma_{x}} B(x, s) \frac{\partial U}{\partial \nu} \Phi(x, s) d s d x \\
\quad=\int_{\Omega} \int_{\Gamma_{x}} B(x, s) \frac{\partial U}{\partial \nu} \varphi(x) d s d x+\int_{\Omega} \int_{\Gamma_{x}} B(x, s) \frac{\partial U}{\partial \nu} s_{3} \vec{\nabla} \varphi(x) \cdot \vec{n}_{x} d s d x \\
\quad=\int_{\Omega}\left\{\int_{\Gamma_{x}} B(x, s) \frac{\partial U}{\partial \nu}(x, s) d s-\vec{\nabla} \cdot\left(\int_{\Gamma_{x}} B(x, s) s_{3} \frac{\partial U}{\partial \nu}(x, s) d s \vec{n}_{x}\right)\right\} \varphi(x) d x .
\end{aligned}
$$


Therefore, we define the source term $Q$ by

$$
Q(x, t) \equiv \int_{\Gamma_{x}} B(x, s) \frac{\partial U}{\partial \nu} d s-\vec{\nabla} \cdot\left(\int_{\Gamma_{x}} B(x, s) s_{3} \frac{\partial U}{\partial \nu} d s \vec{n}_{x}\right),
$$

and this completes the system.

If the cells $\Omega_{x}$ are symmetric about the plane $T_{x}$, then it is easy to separate the effects of storage from those associated with the flux. In order to do this we consider the cell problem $(2.1 \mathrm{~b})$ as two problems, one with symmetric boundary conditions arising from the value of the fissure density, $w(t)=u(x, t)$, and the other having antisymmetric boundary conditions from the first variation of the density, $\vec{w}(t)=\vec{\nabla} u(x, t)$. In this way $(2.1 \mathrm{~b})$ becomes

$$
\begin{cases}\frac{\partial}{\partial t}\left(b U_{1}\right)-\vec{\nabla}_{y} \cdot\left(B \vec{\nabla}_{y} U_{1}\right)=0, & y \in \Omega_{x}, t>0, \\ U_{1}(s, t)=w(t), & s \in \Gamma_{x}\end{cases}
$$

and

$$
\begin{cases}\frac{\partial}{\partial t}\left(b U_{2}\right)-\vec{\nabla}_{y} \cdot\left(B \vec{\nabla}_{y} U_{2}\right)=0, & y \in \Omega_{x}, t>0, \\ U_{2}(s, t)=s_{3} \vec{n}_{x} \cdot \vec{w}(t), & s \in \Gamma_{x} .\end{cases}
$$

Note that $U=U_{1}+U_{2}$ satisfies $(2.1 \mathrm{~b})$ and that $U_{1}$ contributes only to storage while $U_{2}$ is associated with the flux in the $\vec{n}_{x}$ direction, so that, in this case

$$
Q(x, t)=\int_{\Gamma_{x}} B(x, s) \frac{\partial U_{1}}{\partial \nu} d s-\vec{\nabla} \cdot\left(\int_{\Gamma_{x}} s_{3} B(x, s) \frac{\partial U_{2}}{\partial \nu} \vec{n}_{x} d s\right) .
$$

This can furthermore be expressed as a convolution functional of the general input to the cell problem. Thus using Gauss's theorem on (2.2a) yields

$$
\frac{\partial}{\partial t} \int_{\Omega_{x}} b U_{1} d y=\int_{\Gamma_{x}} B \frac{\partial U_{1}}{\partial \nu} d s
$$

and similarly after multiplying $(2.2 \mathrm{~b})$ by $y_{3}$ we get

$$
\frac{\partial}{\partial t} \int_{\Omega_{x}} b U_{2} d y+\int_{\Omega_{x}} B \frac{\partial U_{2}}{\partial y_{3}} d y=\int_{\Gamma_{x}} B \frac{\partial U_{2}}{\partial \nu} s_{3} d s .
$$

Since the input boundary conditions in (2.2) are independent of the spatial variable, one can use standard Green's functions representations for their solutions to express the left side of each of (2.2) as convolution-in-time products, e.g.,

$$
\int_{\Omega_{x}} b(x, y) U_{1}(x, y, t) d y=\int_{0}^{t} k_{1}(x, t-\tau) * w(\tau) d \tau=k_{1}(x, \cdot) * w(t) .
$$

With the obvious notation we obtain by substituting these representations of (2.4) into (2.3) and then into (2.1a) the functional partial differential equation

$$
\begin{aligned}
\frac{\partial}{\partial t}(a(x) & \left.u(x, t)+k_{1}(x, \cdot) * u(x, t)\right) \\
-\vec{\nabla} \cdot & \left(A(x) \vec{\nabla} u(x, t)+\frac{\partial}{\partial t} k_{12}(x, \cdot) * \vec{\nabla} u(x, t)+k_{2}(x, \cdot) * \vec{\nabla} u(x, t)\right) \\
& =f(x, t), \quad x \in \Omega, t>0 .
\end{aligned}
$$


In the case in which $\Omega_{x}$ is independent of $x$, i.e., $\Omega_{x}=\Omega_{0}$ for all $x \in \Omega$, the convolution kernels $k_{1}, k_{12}, k_{2}$ are likewise independent of $x$, and then (2.5) is Nunziato's equation. This was presented in [11] as a generalization of earlier work of Gurtin and Chen for heat conduction models in which the heat flux is permitted to depend on the present value as well as the history of the temperature gradient. No physical or philosophical argument was presented there for this interesting generalization. See [12] for additional work on (2.5).

3. The variational formulation. We will now return to a more general setting and give a mathematical formulation of the microstructure models described above as evolution equations on various Banach spaces. We will show that the CauchyDirichlet problem is well-posed for the quasi-linear parabolic system

$$
\begin{gathered}
\frac{\partial}{\partial t}(a(x) u(x, t))-\vec{\nabla} \cdot A(x, \vec{\nabla} u(x, t))+\int_{\Gamma_{x}} B\left(x, s, \vec{\nabla}_{y} U(x, s, t) \cdot \vec{\nu} d s\right) \\
-\vec{\nabla} \cdot\left(\int_{\Gamma_{x}} B\left(x, s, \vec{\nabla}_{y} U(x, s, t)\right) \cdot \vec{\nu}_{3} \vec{n}_{x} d s\right)=f(x, t), \quad x \in \Omega, \\
\frac{\partial}{\partial t}(b(x, y) U(x, y, t))-\vec{\nabla}_{y} \cdot B\left(x, y, \vec{\nabla}_{y} U(x, y, t)\right) \\
=F(x, y, t), \quad x \in \Omega, y \in \Omega_{x}, \\
-B\left(x, s, \vec{\nabla}_{y} U(x, s)\right) \cdot \vec{\nu}=\mu\left(U(x, s, t)-u(x)-\vec{\nabla} u(x) \cdot \vec{n}_{x} s_{3}\right), \\
x \in \Omega, s=\left(s_{1}, s_{2}, s_{3}\right) \in \Gamma_{x},
\end{gathered}
$$

where $a \in L^{\infty}(\Omega), b \in L^{\infty}\left(\Omega \times \Omega_{x}\right), A$ and $B$ are nonlinear with polynomial growth rates of orders $p-1$ and $q-1$, respectively, $\vec{\nu}$ is the unit outward normal on $\Gamma_{x}$, and $\mu>0$.

Let $\Omega$ be an open, bounded domain in $\mathbb{R}^{3}$, and for every $x \in \Omega$, let $\Omega_{x}$ be a bounded region contained in $\mathbb{R}^{3}$. Identify the product space $\prod_{x \in \Omega} \Omega_{x} \equiv \mathbb{Q}$ as a subset of $\mathbb{R}^{6}$; we require that $\mathbb{Q}$ be a measurable subset of $\mathbb{R}^{6}$, so each of the cells $\Omega_{x} \equiv\{y:(x, y) \in \mathbb{Q}\}$ is a measurable subset of $\mathbb{R}^{3}$. We will further assume that the boundary of $\Omega_{x}$, denoted by $\Gamma_{x}$, is piecewise $C^{1}$ and that the measures $\left|\Gamma_{x}\right|$ and $\left|\Omega_{x}\right|$ are uniformly bounded in $x$. Let a measurable vector field $\vec{n}_{x}$ be defined as in Sec. 2, and for $p, q \geq 2$ define the spaces

$$
W_{0}^{1, p}(\Omega), \quad V_{q}=\left\{U \in L^{2}\left(\Omega, L^{2}\left(\Omega_{x}\right)\right): \vec{\nabla}_{y} U \in L^{q}\left(\Omega, L^{q}\left(\Omega_{x}\right)\right)\right\} .
$$

See [1] for information on Sobolev spaces. To define the norm on $V_{q}$ we will employ the following notation:

$$
\begin{aligned}
|U|_{q} & =\left(\int_{\Omega} \int_{\Omega_{x}}\left|\vec{\nabla}_{y} U(x, y)\right|^{q} d y d x\right)^{1 / q}, \\
\|U\|_{L^{2}(\mathbb{Q})} & =\left(\int_{\Omega} \int_{\Omega_{x}}|U(x, y)|^{2} d y d x\right)^{1 / 2},
\end{aligned}
$$


and $\|U\|_{q}=|U|_{q}+\|U\|_{L^{2}(\mathbb{Q})}$. Denote the product of the Banach spaces $W_{0}^{1, p}(\Omega)$ and $\left(V_{q},\|\cdot\|_{q}\right)$ by $V=W_{0}^{1, p}(\Omega) \times V_{q}$ with norm

$$
\|[u, U]\|_{V}=\|u\|_{W_{0}^{1, p}(\Omega)}+\|U\|_{q} .
$$

For purely notational purposes, we will also define the seminorm space $W_{q}=\left(V_{q},|\cdot|_{q}\right)$.

Let $\gamma_{x}$ be the usual trace map of $W^{1,2}\left(\Omega_{x}\right)$ into $L^{2}\left(\Gamma_{x}\right)$, and define $\mathscr{B}=$ $L^{2}\left(\Omega, L^{2}\left(\Gamma_{x}\right)\right)$ and the distributed trace $\gamma: V_{2} \rightarrow \mathscr{B}$ by $\gamma U(x, s)=\left(\gamma_{x} U(x)\right)(s)$. Since $q \geq 2$, Hölder's inequality and the uniform bound on $\left|\Omega_{x}\right|$ imply that $V_{2}$ is contained in $L^{2}\left(\Omega, W^{1,2}\left(\Omega_{x}\right)\right)$. We will require that the trace maps $\gamma_{x}$ be uniformly bounded. From the above we see that $\gamma$ maps $V_{2}$ into $\mathscr{B}$. Define $\lambda: W_{0}^{1, p}(\Omega) \rightarrow \mathscr{B}$ by

$$
\lambda u(x, s)=u(x) \mathbf{1}_{s}+\vec{\nabla} u(x) \cdot \vec{n}_{x} s_{3}, \quad x \in \Omega, s=\left(s_{1}, s_{2}, s_{3}\right) \in \Gamma_{x},
$$

where $u(x) \mathbf{1}_{s}$ is the constant function on $\Gamma_{x}$ with value $u(x)$. Note that $\vec{\nabla} u \in$ $L^{p}(\Omega)$ and, since $p \geq 2$, we have by Cauchy-Schwartz

$$
\begin{aligned}
& \int_{\Omega} \int_{\Gamma_{x}} \vec{\nabla} u(x) \cdot \vec{n}_{x} s_{3} d s d x \\
& \quad \leq\left(\int_{\Omega} \int_{\Gamma_{x}}\left|\vec{\nabla} u(x) \cdot \vec{n}_{x}\right|^{2} d s d x\right)^{1 / 2}\left(\int_{\Omega} \int_{\Gamma_{x}}\left|s_{3}\right|^{2} d s d x\right)^{1 / 2} \\
& \leq C\left\|\vec{\nabla} u \cdot \vec{n}_{x}\right\|_{L^{2}(\Omega)} \\
& \quad \leq C\left\|\vec{\nabla} u \cdot \vec{n}_{x}\right\|_{L^{p}(\Omega)} .
\end{aligned}
$$

In the remainder of this work we will employ the notation $\tilde{u}=[u, U]$.

Define $V_{h}=\{\tilde{u} \in V: \gamma U=\lambda u$ in $\mathscr{B}\}$. We know that $\gamma$ and $\lambda$ are continuous; so $V_{h}$ is a closed subspace of $V$.

Let $a$ be an element of $L^{\infty}(\Omega)$ such that $a(x) \geq c$ for almost every $x \in \Omega$ and some $c>0$, and let $b$ be an element of $L^{\infty}\left(\Omega \times \Omega_{x}\right)$ such that $b(x, y) \geq c$ for almost every $y \in \Omega_{x}$ and $x \in \Omega$. Define the Hilbert space $H=L^{2}(\Omega) \times$ $L^{2}\left(\Omega, L^{2}\left(\Omega_{x}\right)\right)$ with the inner product

$$
(\tilde{u}, \tilde{\varphi})_{H}=\int_{\Omega} a(x) u(x) \varphi(x) d x+\int_{\Omega} \int_{\Omega_{x}} b(x, y) U(x, y) \Phi(x, y) d y d x,
$$

$$
\text { for } \tilde{u}=[u, U], \tilde{\varphi}=[\varphi, \Phi] \in H .
$$

This is equivalent to the usual inner product when $a=b=1$.

Define $V_{0} \equiv\left\{U \in V_{2}: \gamma U=0\right\}$. We claim that $W_{0}^{1, p}(\Omega) \times V_{0}$ and $V_{h}$ are dense in $H$. To establish the density in the first case we need only show that $V_{0}$ is dense in $L^{2}\left(\Omega, L^{2}\left(\Omega_{x}\right)\right)$. Let $F \in L^{2}\left(\Omega, L^{2}\left(\Omega_{x}\right)\right)$, and define $\theta_{n}$ by

$$
\theta_{n}(x, y)= \begin{cases}1 & \text { if } \inf \left\{|y-z|: z \in \partial \Omega_{x}\right\}>\frac{1}{n}, x \in \Omega, y \in \Omega_{x}, \\ 0 & \text { otherwise }\end{cases}
$$


and $F_{n}$ by

$$
F_{n}(x, y)=\theta_{n}(x, y) F(x, y)
$$

For each $x$ and $n>0$ select mollifier functions $\rho_{m} \in C_{0}^{\infty}\left(B_{1 / m}\right)$ such that

$$
\int_{\mathbb{R}^{N}} \rho_{m}(y) d y=1 \text { and } \rho_{m}(y) \geq 0, y \in B_{1, m}
$$

where $B_{1 / m}=\left\{y \in \mathbb{R}^{N}:|y| \leq 1 / m\right\}$.

Suppressing the (fixed) $x$ write $F_{n}(y)$ for $F_{n}(x, y)$ and define

$$
F_{m, n}(y)=\int_{\Omega_{x}} \rho_{m}(y-z) F_{n}(z) d z
$$

Then $F_{m, n} \in C_{0}^{\infty}\left(\Omega_{x}\right)$ and $\left\|F_{m, n}\right\|_{L^{2}\left(\Omega_{x}\right)} \leq\left\|F_{n}\right\|_{L^{2}\left(\Omega_{x}\right)}$ for a.e. $x \in \Omega$ and $m \geq n$. Also $F_{m, n} \rightarrow F_{n}$ in $L^{2}\left(\Omega_{x}\right)$ as $n \rightarrow \infty$. Clearly, $F_{n} \rightarrow F$ pointwise in $\Omega \times \Omega_{x}$ as $n \rightarrow \infty$. Since $\left|F_{n}(x, y)-F(x, y)\right|^{2} \leq|F(x, y)|^{2}$ and $|F|^{2} \in L^{1}\left(\Omega \times \Omega_{x}\right)$, the Lebesgue dominated convergence theorem implies that $F_{n} \rightarrow F$ in $L^{2}\left(\Omega, L^{2}\left(\Omega_{x}\right)\right)$. For a fixed $M>0, F_{m, M} \rightarrow F_{M}$ in $L^{2}\left(\Omega_{x}\right)$; thus $F_{m, M}-F_{m} \rightarrow 0$ in $L^{2}\left(\Omega, L^{2}\left(\Omega_{x}\right)\right)$ by another application of Lebesgue's theorem. Since $F_{m}$ can be made arbitrarily close to $F$, this proves that $V_{0}$ is dense in $L^{2}\left(\Omega, L^{2}\left(\Omega_{x}\right)\right)$.

To show that $V_{h}$ is dense in $H$, let $[f, F] \in H$ and choose a sequence $\varphi_{n} \epsilon$ $C_{0}^{\infty}(\Omega)$ such that $\varphi_{n} \rightarrow f$ in $L^{2}(\Omega)$ (e.g., by truncation and convolution as above).

For $u \in W^{1, p}(\Omega)$, extend $\lambda u$ to all of $\Omega \times \bigcup_{x \in \Omega} \Omega_{x}$ by

$$
\lambda u(x, y)=u(x) \mathbf{1}_{y}+\vec{\nabla} u(x) \cdot\left(y \cdot \vec{n}_{x}\right) \vec{n}_{x} .
$$

Note that $\gamma(\lambda u)(x, s)=\lambda u(x, s)$. Note also that $\lambda \varphi_{n}$ is in $L^{2}\left(\Omega, L^{2}\left(\Omega_{x}\right)\right)$; thus for every $n>0, F-\lambda \varphi_{n} \in L^{2}\left(\Omega, L^{2}\left(\Omega_{x}\right)\right)$. Fix $n$ and choose $\Psi_{m} \in V_{0}$ such that $\Psi_{m} \rightarrow F-\lambda \varphi_{n}$ in $L^{2}\left(\Omega, L^{2}\left(\Omega_{x}\right)\right)$. Define $\Phi_{m}$ by $\Phi_{m}(x, y)=\Psi_{m}(x, y)+$ $\lambda \varphi_{n}(x, y)$.

For $n>0$ choose $m_{n}>0$ such that $\left\|\Psi_{m_{n}}-\left(F-\lambda \varphi_{n}\right)\right\|_{L^{2}\left(\Omega, L^{2}\left(\Omega_{\gamma}\right)\right)}<1 / n$; then $\left[\varphi_{n}, \Phi_{m_{n}}\right] \rightarrow[f, F]$ in $H$ as $n \rightarrow \infty$. Also $\gamma \Phi_{m_{n}}(x, s)=\gamma \Psi_{m_{n}}(x, s)+$ $\gamma\left(\lambda \varphi_{n}\right)(x, s)=\lambda \varphi_{n}(x, s)$; thus, $\left[\varphi_{n}, \Phi_{m_{n}}\right] \in V_{h}$.

We shall write the system (3.1) as an evolution equation over the spaces described above. To obtain the variational form for the system, choose $[\varphi, \Phi] \in V$, multiply (3.1a) by $\varphi$, and integrate over $\Omega$. Multiply (3.1b) by $\Phi$ and integrate over both 
$\Omega_{x}$ and $\Omega$. Add these equations and apply Green's Theorem to obtain

$$
\begin{aligned}
& \int_{\Omega}\{\left.\frac{\partial}{\partial t}(a(x) u(x, t)) \varphi(x)+\int_{\Omega_{x}} \frac{\partial}{\partial t}(b(x, y) U(x, y, t)) \Phi(x, y) d y\right\} d x \\
& \quad+\int_{\Omega}\left\{A(x, \vec{\nabla} u(x, t)) \cdot \vec{\nabla} \varphi(x)+\int_{\Gamma_{x}} B\left(x, s, \vec{\nabla}_{y} U(x, s, t)\right) \cdot \vec{\nu} \varphi(x) d s\right. \\
& \quad+\int_{\Omega_{x}} B\left(x, y, \vec{\nabla}_{y} U(x, y, t)\right) \cdot \vec{\nabla}_{y} \Phi(x, y) d y \\
&\left.\quad-\int_{\Gamma_{x}} B\left(x, s, \vec{\nabla}_{y} U(x, s, t)\right) \cdot \vec{\nu} \gamma_{x} \Phi(x, s) d s\right\} d x \\
&+\int_{\Omega}\left(\int_{\Gamma_{x}} B\left(x, s, \vec{\nabla}_{y} U(x, s, t)\right) s_{3} \vec{n}_{x} d s\right) \cdot \vec{\nabla} \varphi(x) d x \\
& \quad=\int_{\Omega} f(x) \varphi(x) d x+\int_{\Omega} \int_{\Omega_{x}} F(x, y) \Phi(x, y) d y d x
\end{aligned}
$$

Combining the boundary integrals and substituting for $B\left(x, s, \vec{\nabla}_{y} U(x, y, t)\right) \cdot \vec{\nu}$ yields

$$
\begin{aligned}
& {[u(t), U(t)] \in V: \int_{\Omega} \frac{\partial}{\partial t} a(x) u(x, t) \varphi(x) d x} \\
& \quad+\int_{\Omega} \int_{\Omega_{x}} \frac{\partial}{\partial t} b(x, y) U(x, y, t) \Phi(x, y) d y d x \\
& \quad+\int_{\Omega} A(x, \vec{\nabla} u(x, t)) \cdot \vec{\nabla} \varphi(x) d x \\
& \quad+\int_{\Omega} \int_{\Omega_{x}} B\left(x, y, \vec{\nabla}_{y} U(x, y, t)\right) \cdot \vec{\nabla}_{y} \Phi(x, y) d y d x \\
& +\int_{\Omega} \int_{\Gamma_{x}} \mu(\gamma U(x, s, t)-\lambda u(x, s, t))(\gamma \Phi(x, s, t)-\lambda \varphi(x, s, t)) d s d x \\
& \quad=\int_{\Omega} f(x) \varphi(x) d x+\int_{\Omega} \int_{\Omega_{x}} F(x, y) \Phi(x, y) d y d x, \quad[\varphi, \Phi] \in V .
\end{aligned}
$$

A special case of the above is obtained when (3.1c) is replaced by

$$
\gamma U(x, s, t)=\lambda u(x, s, t), \quad x \in \Omega, s \in \Gamma_{x}, t>0 .
$$

This is the formal result obtained by setting $\mu=\frac{1}{\delta}$ and allowing $\delta \rightarrow 0^{+}$, so that 
(3.1c) ${ }^{\prime}$ is forced to hold and corresponds to

$$
\begin{aligned}
& {[u(t), U(t)] \in V_{h}: \int_{\Omega} \frac{\partial}{\partial t} a(x) u(x, t) \varphi(x) d x} \\
& \quad+\int_{\Omega} \int_{\Omega_{x}} \frac{\partial}{\partial t} b(x, y) U(x, y) \Phi(x, y) d y d x \\
& \quad+\int_{\Omega} A(x, \vec{\nabla} u(x, t)) \cdot \vec{\nabla} \varphi(x) d x \\
& \quad+\int_{\Omega} \int_{\Omega_{x}} B\left(x, y, \vec{\nabla}_{x} U(x, y, t)\right) \cdot \vec{\nabla}_{y} \Phi(x, y) d y d x \\
& \quad=\int_{\Omega} f(x) \varphi(x) d x+\int_{\Omega} \int_{\Omega_{x}} F(x, y) \Phi(x, y) d y d x, \quad[\varphi, \Phi] \in V_{h} .
\end{aligned}
$$

Set $\mu_{\delta}=\frac{1}{\delta}$; then (3.1) with $\mu_{\delta}$ in place of $\mu$ will be called the regularized model and $(3.1)^{\prime}$, i.e., (3.1a), (3.1b), and (3.1c) $)^{\prime}$, will be called the matched model. Conversely, starting from (3.2) it is not difficult to recover (3.1).

Define the Banach spaces

$$
\begin{aligned}
& \mathscr{H}=L^{2}(0, T ; H), \\
& \mathscr{W}_{p}=L^{p}\left(0, T ; W_{0}^{1, p}(\Omega)\right), \\
& \mathscr{V}_{q}=L^{q}\left(0, T ; V_{q}\right), \\
& \mathscr{V}=\mathscr{W}_{p} \times \mathscr{V}_{q}, \\
& \mathscr{V}_{h}=\{\tilde{u} \in \mathscr{V}: \gamma U(t)=\lambda u(t) \text { in } \mathscr{B} \text { for almost every } t>0\} .
\end{aligned}
$$

Let $A: \Omega \times \mathbb{R}^{3} \rightarrow \mathbb{R}^{3}$ satisfy the following:

$A(x, \vec{\xi})$ is continuous in $\vec{\xi} \in \mathbb{R}^{3}$ and measurable in $x$ with

$$
|A(x, \vec{\xi})| \leq C|\vec{\xi}|^{p-1}+g_{1}(x)
$$

where $g_{1}$ is given in $L^{p^{\prime}}(\Omega), C$ and $c>0$.

$\langle A(x, \vec{\xi})-A(x, \vec{\eta}), \vec{\xi}-\vec{\eta}\rangle \geq 0$ for $\vec{\xi}, \vec{\eta} \in \mathbb{R}^{3}$ for almost every $x \in \Omega$.

$A(x, \vec{\xi}) \cdot \vec{\xi} \geq c|\vec{\xi}|^{p}$ for almost every $x \in \Omega$ and all $\vec{\xi} \in \mathbb{R}^{3}$.

Let $B: \mathbb{Q} \times \mathbb{R}^{3} \rightarrow \mathbb{R}^{3}$ satisfy:

$B(x, y, \vec{\xi})$ is continuous in $\vec{\xi}$ and measurable in $x$ and $y$ with

$$
|B(x, y, \vec{\xi})| \leq C|\vec{\xi}|^{q-1}+h_{1}(x, y)
$$

where $h_{1} \in L^{q^{\prime}}(\mathbb{Q})$.

$$
\langle B(x, y, \vec{\xi})-B(x, y, \vec{\eta}), \vec{\xi}-\vec{\eta}\rangle \geq 0, \quad \vec{\xi}, \vec{\eta} \in \mathbb{R}^{n},
$$

for almost every $x \in \Omega, y \in \Omega_{x}$.

$B(x, y, \vec{\xi}) \cdot \vec{\xi} \geq c|\vec{\xi}|^{q}$ for almost all $x \in \Omega, y \in \Omega_{x}$, and all $\vec{\xi} \in \mathbb{R}^{n}$. 
Define $L: V \rightarrow V^{\prime}$ by

$L \tilde{u}(\tilde{\varphi})=\int_{\Omega} A(x, \vec{\nabla} u) \cdot \vec{\nabla} \varphi d x+\int_{\Omega} \int_{\Omega_{x}} B(x, y, \vec{\nabla} U) \cdot \vec{\nabla}_{y} \Phi d y d x, \quad \tilde{u}, \tilde{\varphi} \in V$,

where $A$ and $B$ are as described.

The conditions above on $A$ and $B$ lead to estimates on the operator $L$ of the types

$$
L \tilde{u}(\tilde{u}) \geq c\left\{\int_{\Omega}|\vec{\nabla} u|^{p} d x+\int_{\Omega} \int_{\Omega_{x}}|\vec{\nabla} U|^{q} d y d x\right\}
$$

and

$$
\begin{aligned}
L \tilde{u}(\tilde{\varphi}) \leq & \int_{\Omega}|A(x, \vec{\nabla} u)||\vec{\nabla} \varphi| d x+\int_{\Omega} \int_{\Omega_{x}}\left|B\left(x, y, \vec{\nabla}_{y} U\right)\right|\left|\vec{\nabla}_{y} \Phi(x, y)\right| d y d x \\
\leq & \left\{C\|\vec{\nabla} u\|_{L^{p}(\Omega)}^{p-1}+\|g\|_{L^{p^{\prime}}(\Omega)}\right\}\|\vec{\nabla} \varphi\|_{L^{p}(\Omega)} \\
& +\left\{C\left\|\vec{\nabla}_{y} U\right\|_{L^{q}\left(\Omega, L^{q}\left(\Omega_{x}\right)\right)}^{q-1}+\left\|h_{1}\right\|_{L^{q^{\prime}}\left(\Omega, L^{q^{\prime}}\left(\Omega_{x}\right)\right)}\right\}\|\vec{\nabla} \Phi\|_{L^{q}\left(\Omega, L^{q}\left(\Omega_{x}\right)\right)}
\end{aligned}
$$

thus,

$$
L \tilde{u}(\tilde{\varphi}) \leq C\left(\|u\|_{W_{0}^{1, p}}^{p-1}+\|U\|_{q}^{q-1}+k\right)\|\tilde{\varphi}\|_{V}, \quad \tilde{u}, \tilde{\varphi} \in V ;
$$

so $L$ is bounded from $V$ into $V^{\prime}$.

Define $M: \mathscr{B} \rightarrow \mathscr{B}^{\prime}$ by

$$
M([\Phi, \Psi])=\int_{\Omega} \int_{\Gamma_{x}} \Phi \Psi d s d x, \quad \Phi, \Psi \in \mathscr{B} .
$$

Denote the action of the pair $[\lambda, \gamma]: V \rightarrow \mathscr{B}$ by $[\lambda, \gamma](u, U)=\gamma U-\lambda u$. Then

$$
\begin{aligned}
\mathscr{M}(\tilde{u}, \tilde{\varphi}) & \equiv[\lambda, \gamma]^{*} \circ M \circ[\lambda, \gamma](\tilde{u})(\tilde{\varphi}) \\
& =\int_{\Omega} \int_{\Gamma_{x}}(\gamma U-\lambda u)(\gamma \Phi-\lambda \varphi) d s d x, \quad \tilde{u}, \tilde{\varphi} \in V,
\end{aligned}
$$

gives a continuous and linear function $\mathscr{M}: V \rightarrow V^{\prime}$.

Define $\tilde{f} \in V^{\prime}$ by

$$
\tilde{f}(\tilde{\varphi})=\int_{\Omega} f \varphi d x+\int_{\Omega} \int_{\Omega_{x}} F \Phi d y d x, \quad \tilde{\varphi} \in V .
$$

We will use the same notation to refer to the corresponding realizations of these operators on the spaces $\mathscr{H}, \mathscr{V}$, and $\mathscr{V}_{h}$.

Integrating (3.2) from 0 to $T$ we obtain

$$
\begin{gathered}
\tilde{u} \in \mathscr{V}: \int_{0}^{T}\left(\frac{\partial}{\partial t} \tilde{u}, \tilde{\varphi}\right)_{H} d t+\int_{0}^{T} L \tilde{u}(\tilde{\varphi}) d t+\int_{0}^{T} \frac{1}{\delta} \mathscr{M} \tilde{u}(\tilde{\varphi}) d t \\
=\int_{0}^{T} \tilde{f}(\tilde{\varphi}) d t, \quad \tilde{\varphi} \in \mathscr{V} .
\end{gathered}
$$

Similarly from $(3.2)^{\prime}$ we get

$$
\tilde{u} \in \mathscr{V}_{h}: \int_{0}^{T}\left(\frac{\partial}{\partial t} \tilde{u}, \tilde{\varphi}\right)_{H} d t+\int_{0}^{T} L \tilde{u}(\tilde{\varphi}) d t=\int_{0}^{T} \tilde{f}(\tilde{\varphi}) d t, \quad \tilde{\varphi} \in \mathscr{V}_{h} .
$$


We shall give conditions under which (3.5) and (3.5) have unique solutions and show that the solutions $\tilde{u}_{\delta}$ of (3.5) converge to the solution $\tilde{u}$ of $(3.5)^{\prime}$ as $\delta \rightarrow 0$. This is our main result, and we prove it in the following section.

THEOREM. Given the spaces and operators as above, suppose that $\tilde{u}_{0}=\left[u_{0}, U_{0}\right] \in H$ and $\tilde{f}=[f, F] \in \mathscr{V}^{\prime}$. Then for every $\delta>0$ there is a unique $\tilde{u}_{\delta} \in \mathscr{V}$ which satisfies $(3.5)$ and $\tilde{u}_{\delta}(0)=\tilde{u}_{0}$. Also, there is a unique $\tilde{u} \in \mathscr{V}_{h}$ which satisfies (3.5) and where $\tilde{u}(0)=\tilde{u}_{0}$. Furthermore, $\tilde{u}_{\delta}$ converges weakly to $\tilde{u}$ in $\mathscr{V}$ as $\delta \rightarrow 0$.

4. The proofs. We will need the following standard result.

Lemma. If $\tilde{u}$ is an element of $\mathscr{V}$ such that (3.5) or $(3.5)^{\prime}$ holds, then $|\tilde{u}(\cdot)|_{H}^{2}$ is absolutely continuous on $[0, T]$ and

$$
\frac{d}{d t}|\tilde{u}(t)|_{H}^{2}=2\left\langle\tilde{u}^{\prime}(t), \tilde{u}(t)\right\rangle \text { for almost every } t \in[0, T] \text {. }
$$

This follows since $H$ is the pivot space between $\mathscr{V}$ and $\mathscr{V}^{\prime}$ and between $\mathscr{V}_{h}$ and $\mathscr{V}_{h}^{\prime}$.

Definition. Let $V$ be a reflexive Banach space and $\mathscr{A}: V \rightarrow V^{\prime}$. Then $\mathscr{A}$ is said to be type-M if

(i) $u_{n} \rightarrow u$ weakly in $V$,

(ii) $\mathscr{A} u_{n} \rightarrow f$ weakly in $V^{\prime}$, and

(iii) $\quad \limsup _{n \rightarrow \infty} \mathscr{A} u_{n}\left(u_{n}\right) \leq f(u)$

imply that $\mathscr{A} u=f$.

Some combinations of properties will imply that a function $\mathscr{A}$ is type- $M$. For example, any monotone and continuous function $\mathscr{A}: V \rightarrow V^{\prime}$ is type-M (see [10]).

Integrating (3.4) from 0 to $T$ and applying Hölder's inequality once more shows that $L$ is bounded from $\mathscr{V}$ into $\mathscr{V}^{\prime}$. (We will use the same notation for both situations; $L: V \rightarrow V^{\prime}$ and $L: \mathscr{V} \rightarrow \mathscr{V}^{\prime}$.) The Caratheodory-type conditions prescribed above on $\mathscr{A}$ and $\mathscr{B}$ guarantee that $L$ is both continuous and monotone. Since $\mathscr{M}$ is also continuous and monotone, both $L$ and $L+\frac{1}{\delta} \mathscr{M}$ are type- $M$ from $V$ to $V^{\prime}$ as are their realizations from $\mathscr{V}$ to $\mathscr{V}^{\prime}$.

To establish the existence of a solution to (3.5) or $(3.5)^{\prime}$ we will require some ellipticity estimate on the operator $L$. (Our preceding estimate, (3.3), is not sufficient.) In order to provide such an estimate, we will make a change of variable. This will yield equations equivalent to (3.5) and $(3.5)^{\prime}$ for which we can establish an ellipticity estimate.

Suppose that we are given a $\tilde{u} \in \mathscr{V}$ such that

$$
\left(\frac{\partial}{\partial t} \tilde{u}, \tilde{\varphi}\right)_{H}+L \tilde{u}(\tilde{\varphi})+\frac{1}{\delta} \mathscr{M} \tilde{u}(\tilde{\varphi})=\tilde{f}(\tilde{\varphi}), \quad \tilde{\varphi} \in \mathscr{V} .
$$

Let $\tilde{v}=e^{-t} \tilde{u}$. Substituting for $\tilde{u}$ we obtain:

$$
\left(\left(\frac{\partial}{\partial t} \tilde{v} e^{t}+\tilde{v} e^{t}\right), \tilde{\varphi}\right)_{H}+L\left(\tilde{v} e^{t}\right) \tilde{\varphi}+\frac{1}{\delta} \mathscr{M}\left(\tilde{v} e^{t}\right)(\tilde{\varphi})=\tilde{f}(\tilde{\varphi}), \quad \tilde{\varphi} \in \mathscr{V}
$$


Multiplying by $e^{-t}$ yields

$$
\left(\frac{\partial}{\partial t} \tilde{v}, \tilde{\varphi}\right)_{H}+(\tilde{v}, \tilde{\varphi})_{H}+e^{-2 t} L\left(\tilde{v} e^{t}\right)\left(\tilde{\varphi} e^{t}\right)+\frac{1}{\delta} \mathscr{M}(\tilde{v})(\tilde{\varphi})=e^{-t} \tilde{f}(\tilde{\varphi}), \quad \tilde{\varphi} \in \mathscr{V} .
$$

Define $\mathscr{L}: \mathscr{V} \rightarrow \mathscr{V}^{\prime}$ by

$$
\mathscr{L} \tilde{v}(t)(\tilde{\varphi}(t))=e^{-2 t} L\left(e^{t} \tilde{v}(t)\right)\left(e^{t} \tilde{\varphi}(t)\right)+(\tilde{v}, \tilde{\varphi})_{H}, \quad \tilde{\varphi} \in \mathscr{V},
$$

and define $g \in \mathscr{V}^{\prime}$ by $\tilde{g}(\tilde{\varphi})=e^{-t} \tilde{f}(\tilde{\varphi}), \varphi \in \mathscr{V}$. Thus $\tilde{u}$ solves (4.1) iff

$$
\tilde{v} \in \mathscr{V}:\left(\frac{\partial}{\partial t} \tilde{v}, \tilde{\varphi}\right)_{H}+\mathscr{L} \tilde{v}(\tilde{\varphi})+\frac{1}{\delta} \mathscr{M} \tilde{v}(\tilde{\varphi})=\tilde{g}(\tilde{\varphi}), \quad \tilde{\varphi} \in \mathscr{V},
$$

where $\tilde{v}=e^{-t} \tilde{u}$.

For convenience we will write $\mathscr{L}$ as the sum of three operators. Define $\mathscr{L}_{p}$ :

$$
\begin{aligned}
W_{0}^{1, p}(\Omega) \rightarrow W^{-1, p^{\prime}}(\Omega) & , \mathscr{L}_{q}: V_{q} \rightarrow V_{q}^{\prime} \text {, and } \mathscr{L}_{2}: L^{2}(\mathbb{Q}) \rightarrow L^{2}(\mathbb{Q}) \text { by } \\
\mathscr{L}_{p} u(\varphi) & =e^{-2 t} \int_{\Omega} A\left(x, e^{t} \vec{\nabla} u\right) \cdot \vec{\nabla} \varphi e^{t} d x, \\
\mathscr{L}_{q} U(\Phi) & =e^{-2 t} \int_{\Omega} \int_{\Omega_{x}} B\left(x, y, e^{t} \vec{\nabla}_{y} U\right) \cdot \vec{\nabla} \Phi e^{t} d y d x, \\
\mathscr{L}_{2} \tilde{u}(\tilde{\varphi}) & =(\tilde{u}, \tilde{\varphi})_{H} .
\end{aligned}
$$

Then $\mathscr{L} \tilde{u}(\tilde{\varphi})=\mathscr{L}_{p} u(\varphi)+\mathscr{L}_{q}(U)(\Phi)+\mathscr{L}_{2} \tilde{u}(\tilde{\varphi})$. We will follow the convention established above and use the same notation for the corresponding operators on the spaces $\mathscr{W}_{p}$ and $\mathscr{V}_{q}$.

Note that from the lower estimate on $B$,

$$
\mathscr{L}_{p} u(u) \geq c \int_{\Omega}|\vec{\nabla} u|^{p} d x
$$

and since $u \in W_{0}^{1, p}(\Omega)$ this is equivalent to

$$
\mathscr{L}_{p} u(u) \geq c\|u\|_{W_{0}^{1, p}(\Omega)}^{p}, \quad u \in W_{0}^{1, p}(\Omega) .
$$

Also,

$$
\mathscr{L}_{q}(U)(U) \geq k \int_{\Omega} \int_{\Omega_{x}}\left|\vec{\nabla}_{y} U\right|^{q} d y d x=k|U|_{q}^{q}, \quad U \in V_{q},
$$

and

$$
\mathscr{L}_{2} \tilde{u}(\tilde{u}) \geq \alpha\|U\|_{L^{2}(\mathbb{Q})}^{2},
$$

where $\alpha>0$ depends on $b$. Also for $0 \leq t \leq T$, an estimate similar to (3.4) holds. We have

$$
\mathscr{L} \tilde{u}(\tilde{\varphi}) \leq C\left(\|u\|_{W_{0}^{1, p}(\Omega)}^{p-1}+|U|_{q}^{q-1}+\|U\|_{L^{2}(\mathbb{Q})}+k\right)\|\tilde{\varphi}\|_{V}
$$

for $\tilde{u}, \tilde{\varphi} \in V$. Thus $\mathscr{L}$ is bounded from $V$ into $V^{\prime} . \mathscr{L}$ also shares the other properties of $L$; it and $\mathscr{L}+\frac{1}{\delta} \mathscr{M}$ are continuous and monotone and thus type- $M$ from $V$ into $V^{\prime}$ and $\mathscr{V}$ into $\mathscr{V}^{\prime}$. 
We shall next establish existence and uniqueness of functions $\tilde{u}_{\delta} \in \mathscr{V}$ and $\tilde{u} \in \mathscr{V}_{h}$ such that

$$
\begin{aligned}
\int_{0}^{T} & \left(\frac{d}{d t} \tilde{u}_{\delta}, \tilde{\varphi}\right)_{H} d t+\int_{0}^{T} \mathscr{L} \tilde{u}_{\delta}(\tilde{\varphi}) d t+\frac{1}{\delta} \int_{0}^{T} \mathscr{M} \tilde{u}_{\delta}(\tilde{\varphi}) d t \\
& =\int_{0}^{t} \tilde{f}(\tilde{\varphi}) d t, \quad \tilde{\varphi} \in \mathscr{V},
\end{aligned}
$$

and

$$
\int_{0}^{T}\left(\frac{d}{d t} \tilde{u}, \tilde{\varphi}\right)_{H} d t+\int_{0}^{T} \mathscr{L} \tilde{u}(\tilde{\varphi}) d t=\int_{0}^{T} \tilde{f}(\tilde{\varphi}) d t, \quad \tilde{\varphi} \in \mathscr{V}_{h},
$$

where $\tilde{u}(0)=\tilde{u}_{\delta}(0)=\tilde{u}_{0} \in H$ and $\tilde{f} \in \mathscr{V}^{\prime}$ are given. By applying a change of variable as above, the existence of unique solutions of $(3.5)$ and $(3.5)^{\prime}$ is then established. The plan is to use a Galerken-type method: we will first solve the problem on subspaces of $V$ and then show that the solutions obtained on successive subspaces converge to the desired solution.

Let $\left\{w_{j}\right\}$ be a basis for $V$ and let $V_{m}$ denote the linear span of $\left\{w_{1}, w_{2}, \ldots, w_{m}\right\}$. Since $\mathscr{L}$ and $\mathscr{L}+\frac{1}{\delta} \mathscr{M}$ from $V$ to $V^{\prime}$ are continuous, their restrictions to the finitedimensional space $V_{m}$ are continuous.

Fix $\delta>0$. Then for each $m \geq 1$, by the Cauchy-Peano Theorem in $V_{m}^{\prime}$, there is a solution (depending on $\delta$ ):

$$
\begin{array}{r}
\tilde{u}_{m} \in V_{m}:\left(\tilde{u}_{m}^{\prime}(t), w_{j}\right)_{H}+\mathscr{L} \tilde{u}_{m}(t)\left(w_{j}\right)+\frac{1}{\delta} \mathscr{M} \tilde{u}_{m}(t)\left(w_{j}\right) \\
=\tilde{f}\left(w_{j}\right), \quad 1 \leq j \leq m, \quad 0 \leq t \leq T_{m} \leq T,
\end{array}
$$

with $\tilde{u}_{m}(0)=u_{m}^{0} \in V_{m}$ where $\tilde{u}_{m}^{0} \rightarrow \tilde{u}_{0}$ in $H$ as $m \rightarrow \infty$. We may replace $w_{j}$ by any element of $V_{m}$. Thus $\tilde{u}_{m}$ satisfies

$$
\begin{gathered}
\tilde{u}_{m} \in V_{m}: \frac{1}{2}\left|\tilde{u}_{m}(t)\right|_{H}^{2}+\int_{0}^{t} \mathscr{L} \tilde{u}_{m}\left(\tilde{u}_{m}\right) d s+\frac{1}{\delta} \int_{0}^{t} \mathscr{M} \tilde{u}_{m}\left(\tilde{u}_{m}\right) d s \\
=\int_{0}^{t} \tilde{f} \tilde{u}_{m} d s+\frac{1}{2}\left|u_{m}(0)\right|_{H}^{2} .
\end{gathered}
$$

We showed previously that for some $\alpha_{1} \in \mathbb{R}$

$$
\mathscr{L}_{p} u_{m}\left(u_{m}\right) \geq \alpha_{1} \int_{\Omega}\left|\vec{\nabla} u_{m}\right|^{p} d x, \quad u_{m} \in W_{0}^{1, p}(\Omega) .
$$

This is equivalent to

$$
\mathscr{L}_{p}\left(u_{m}\right)\left(u_{m}\right) \geq \alpha_{1}\left\|u_{m}\right\|_{W_{0}^{1, p}(\Omega)}^{p}, \quad u_{m} \in W_{0}^{1, p}(\Omega),
$$

for some $\alpha_{1} \in \mathbb{R}$ since $u_{m}$ vanishes on the boundary of $\Omega$. We also showed that for some $\alpha_{2} \in \mathbb{R}, \mathscr{L}_{q} U_{m}\left(U_{m}\right) \geq \alpha_{2}\left|U_{m}\right|_{q}^{q}$ for $U_{m} \in V_{q}$. These two inequalities, the monotonicity of $\mathscr{M}$, and (4.5) combine to yield

$$
\begin{gathered}
\frac{1}{2}\left|\tilde{u}_{m}(t)\right|_{H}^{2}+\alpha_{1} \int_{0}^{t}\left\|u_{m}\right\|_{W_{0}^{1, p}(\Omega)}^{p} d s+\alpha_{2} \int_{0}^{t}\left|U_{m}\right|_{q}^{q} d s+\int_{0}^{t}\left\|U_{m}\right\|_{L^{2}(\mathbb{Q})}^{2} d s \\
\leq \int_{0}^{t} \tilde{f}(s) \tilde{u}(s) d s+\frac{1}{2}\left|\tilde{u}_{m}^{0}\right|_{H}^{2}, \quad 0 \leq t \leq T_{m} .
\end{gathered}
$$


Since the right-hand side of (4.6) is bounded for $0 \leq t \leq T$, the solution $u_{m}$ is bounded in $H$ for $0 \leq t \leq T$; thus we may assume that $T_{m}=T$ for $m \geq 1$.

We may write

$$
\int_{0}^{t} \tilde{f} \tilde{u}_{m} d s=\int_{0}^{t} f u_{m} d s+\int_{0}^{t} F_{1} U_{m} d s+\int_{0}^{t} F_{2} U_{m} d s, \quad 0 \leq t \leq T,
$$

where $F_{1} \in L^{q^{\prime}}\left(0, T ; V_{q}^{\prime}\right), F_{2} \in L^{2}\left(0, T ; L^{2}(\mathbb{Q})\right)$, and $F_{1}+F_{2}=F$. Thus we obtain

$$
\begin{aligned}
\int_{0}^{t} \tilde{f} \tilde{u}_{m} d s \leq & \int_{0}^{t}\|f(x)\|_{W_{0}^{1, p}\left(\Omega^{\prime}\right)}\left\|u_{m}(s)\right\|_{W_{0}^{1, p}(\Omega)} d s+\int_{0}^{t}\left\|F_{1}(s)\right\|_{W_{q}^{\prime}}\left|U_{m}(s)\right|_{q} d s \\
& +\int_{0}^{t}\left\|F_{2}(s)\right\|_{L^{2}(\mathbb{Q})}\left\|U_{m}(s)\right\|_{L^{2}(\mathbb{Q})} d s, \quad 0 \leq t \leq T .
\end{aligned}
$$

Applying Young's inequality to each of the three terms on the right-hand side of the above yields

$$
\begin{aligned}
\int_{0}^{t} \tilde{f} \tilde{u}_{m} d s \leq & \frac{\varepsilon_{1}^{1-p^{\prime}}}{p^{\prime}} \int_{0}^{t}\|f\|_{W_{0}^{1, p}(\Omega)^{\prime}}^{p^{\prime}} d s+\frac{\varepsilon_{1}^{p}}{p} \int_{0}^{t}\left\|u_{m}\right\|_{W_{0}^{1, p}(\Omega)}^{p} d s \\
& +\frac{\varepsilon_{2}^{1-q^{\prime}}}{q^{\prime}} \int_{0}^{t}\left\|F_{1}\right\|_{W_{q}^{\prime}}^{q^{\prime}} d s+\frac{\varepsilon_{2}^{q}}{q} \int_{0}^{t}\left|U_{m}\right|_{q}^{q} d s \\
& +\frac{\varepsilon_{3}^{-1}}{2} \int_{0}^{t}\left\|F_{2}\right\|_{L^{2}(\mathbb{Q})}^{2} d s+\frac{\varepsilon_{3}^{2}}{2} \int_{0}^{t}\left\|U_{m}\right\|_{L^{2}(\mathbb{Q})}^{2} d s, \quad 0 \leq t \leq T,
\end{aligned}
$$

for any $\varepsilon_{1}, \varepsilon_{2}, \varepsilon_{3}>0$. Choose $\varepsilon_{1}=\left(\alpha_{1} p / 2\right)^{1 / p}, \varepsilon_{2}=\left(\alpha_{2} q / 2\right)^{1 / p}$, and $\varepsilon_{3}=1$; then (4.6) and (4.7) give

$$
\begin{gathered}
\frac{1}{2}\left|\tilde{u}_{m}(t)\right|_{H}^{2}+\frac{\alpha_{1}}{2} \int_{0}^{t}\left\|u_{m}\right\|_{W_{0}^{1, p}(\Omega)}^{p} d s+\frac{\alpha_{2}}{2} \int_{0}^{t}\left|U_{m}\right|_{q}^{q} d s \\
+\frac{1}{2} \int_{0}^{t}\left\|U_{m}\right\|_{L^{2}(\mathbb{Q})}^{2} d s \leq C, \quad 0 \leq t \leq T,
\end{gathered}
$$

where $C$ depends only on $\tilde{f}$ and $u_{m}^{0}$. The above inequality shows that $\tilde{u}_{m}$ is bounded in both $\mathscr{V}$ and $L^{\infty}(0, T ; H)$. There is a subsequence, which will be denoted simply by $\tilde{u}_{m}$, such that $\tilde{u}_{m} \rightarrow \tilde{u}$ weakly in $\mathscr{V}, \mathscr{L} \tilde{u}_{m}+\frac{1}{\delta} \mathscr{M}_{m}-\xi$ weakly in $\mathscr{V}^{\prime}$, and $\tilde{u}_{m}(T)-\tilde{u}_{T}$ in $H$.

For each $j \geq 1, \delta>0$, and $\varphi \in C^{1}[0, T]$ we obtain from (4.4)

$$
\begin{aligned}
\tilde{u}_{m} \in V_{m}:-\int_{0}^{T}\left(\tilde{u}_{m}(t), \varphi^{\prime}(t) w_{j}\right)_{H} d t+\int_{0}^{T} L \tilde{u}_{m}(t)\left(\varphi(t) w_{j}\right) d t \\
\quad+\int_{0}^{T} \frac{1}{\delta} \tilde{M}_{m}(t)\left(\varphi(t) w_{j}\right) d t \\
\quad=\int_{0}^{T} \tilde{f}(t) \varphi(t) w_{j} d t+\left(u_{m}^{0}, w_{j}\right) \varphi(0)-\left(u_{m}(T), w_{j}\right) \varphi(T) .
\end{aligned}
$$


Letting $m \rightarrow \infty$ gives

$$
\begin{aligned}
-\int_{0}^{T} & \left(\tilde{u}(t), \varphi^{\prime}(t) w_{j}\right)_{H} d t+\int_{0}^{T} \xi(t) \varphi(t) w_{j} d t \\
& =\left(\tilde{u}_{0}, w_{j}\right) \varphi(0)-\left(\tilde{u}_{T}, w_{j}\right) \varphi(T)+\int_{0}^{T} \tilde{f}(t) \tilde{u}(t) d t .
\end{aligned}
$$

Applying Green's Theorem in the $t$ variance yields

$$
\begin{array}{r}
\int_{0}^{T}\left(\tilde{u}^{\prime}(t), \varphi(t) w_{j}\right)_{H} d t+\int_{0}^{T} \xi(t) \varphi(t) w_{j} d t+\int_{0}^{T}\left(\tilde{u}(t), \varphi(t) w_{j}\right)_{H} d t \\
\quad=\int_{0}^{t} \tilde{f}(t) \tilde{u}(t) d t+\left(\tilde{u}^{0}-\tilde{u}(0), w_{j}\right) \varphi(0)+\left(\tilde{u}(T)-\tilde{u}_{T}, w_{j}\right) \varphi(T) .
\end{array}
$$

Since $\left\{w_{j}\right\}$ is dense in $V$, it follows that

$$
\begin{gathered}
\tilde{u} \in \mathscr{V}: \tilde{u}^{\prime}+\xi=\tilde{f} \quad \text { in } \mathscr{V}^{\prime}, \\
\tilde{u}(0)=\tilde{u}_{0} \text { and } \tilde{u}(T)=\tilde{u}_{T} \text { in } H .
\end{gathered}
$$

We will now show that $\xi=\mathscr{L} \tilde{u}+\frac{1}{\delta} \mathscr{M} \tilde{u}$. From (4.4) we obtain

$$
\int_{0}^{T} \mathscr{L} \tilde{u}_{m}\left(\tilde{u}_{m}\right) d t+\int_{0}^{T} \frac{1}{\delta} \mathscr{M} \tilde{u}_{m}\left(\tilde{u}_{m}\right) d t=\int_{0}^{T} \tilde{f} \tilde{u} d t+\frac{1}{2}\left(\left\|\tilde{u}_{m}^{0}\right\|_{H}^{2}-\left\|\tilde{u}_{m}(T)\right\|_{H}^{2}\right) .
$$

Taking the limsup of the above as $n \rightarrow \infty$, the weak lower semicontinuity of the $H$-norm yields

$$
\limsup _{m \rightarrow \infty} \int_{0}^{T}\left\{\mathscr{L} \tilde{u}_{m}\left(\tilde{u}_{m}\right)+\frac{1}{\delta} \mathscr{M} \tilde{u}_{m}\left(\tilde{u}_{m}\right)\right\} d t \leq \int_{0}^{T} \tilde{f} \tilde{u} d t+\frac{1}{2}\left\|\tilde{u}^{0}\right\|_{H}^{2}-\frac{1}{2}\left\|\tilde{u}_{T}\right\|^{2},
$$

and from (4.9) we have

$$
\left\{\frac{1}{2}\left\|\tilde{u}_{T}\right\|_{H}^{2}-\frac{1}{2}\left\|\tilde{u}_{0}\right\|_{H}^{2}\right\}+\int_{0}^{T} \xi \tilde{u} d t=\int_{0}^{T} \tilde{f} \tilde{u} d t .
$$

Substituting for $\int_{0}^{T} \tilde{f} \tilde{u} d t$ in (8.7) we finally obtain

$$
\limsup _{m \rightarrow \infty} \int_{0}^{T}\left\{\mathscr{L} \tilde{u}_{m}\left(\tilde{u}_{m}\right)+\frac{1}{\delta} \mathscr{M}_{m}\left(\tilde{u}_{m}\right)\right\} d t \leq \int_{0}^{T} \tilde{f} \tilde{u} d t .
$$

Since $\mathscr{L}+\frac{1}{\delta} \mathscr{M}$ is type- $M$, this shows that $\mathscr{L} \tilde{u}+\frac{1}{\delta} \mathscr{M} \tilde{u}=\xi$.

Thus far, we have established the existence of a solution of (4.2) for every $\delta>0$. Suppose that two such solutions $u_{1}$ and $u_{2}$ existed. Writing (4.2) twice, once with $u_{1}$ and again with $u_{2}$, and subtracting yields

$\int_{0}^{T}\left(\frac{d}{d s}\left(\tilde{u}_{1}-\tilde{u}_{2}\right), \tilde{\varphi}\right)_{H}+\int_{0}^{T}\left(\mathscr{L} \tilde{u}_{1}-\mathscr{L} \tilde{u}_{2}\right) \tilde{\varphi} d s \frac{1}{\delta} \int_{0}^{T} \mathscr{M}\left(\tilde{u}_{1}-\tilde{u}_{2}\right) \tilde{\varphi} d s=0, \quad \tilde{\varphi} \in \mathscr{V}$.

Replacing $\tilde{\varphi}(s)$ with

$$
\left(\tilde{u}_{1}-\tilde{u}_{2}\right) \mathbf{1}_{[0, t)}(s)= \begin{cases}\tilde{u}_{1}(s)-\tilde{u}_{2}(s), & 0 \leq s \leq t \\ 0, & s>t\end{cases}
$$


we obtain

$$
\begin{aligned}
& \frac{1}{2}\left\|\tilde{u}_{1}(t)-\tilde{u}_{2}(t)\right\|_{H}^{2}+\int_{0}^{t}\left(\mathscr{L} \tilde{u}_{1}-\mathscr{L} \tilde{u}_{2}\right)\left(\tilde{u}_{1}-\tilde{u}_{2}\right) d s \\
& \quad+\frac{1}{\delta} \int_{0}^{t} \mathscr{M}\left(\tilde{u}_{1}-\tilde{u}_{2}\right)\left(\tilde{u}_{1}-\tilde{u}_{2}\right) d s=0, \quad 0 \leq t \leq T .
\end{aligned}
$$

Since $\mathscr{L}$ and $\frac{1}{\delta} \mathscr{M}$ are monotone, this shows that $\left\|\tilde{u}_{1}(t)-\tilde{u}_{2}(t)\right\|_{H}^{2}=0$; thus, $u_{1}=u_{2}$.

We have shown that for every $\delta>0$ there is a unique solution to (4.2). Existence and uniqueness of a solution to (4.3) is shown in the same way. The variational statements are the same, but since $\mathscr{M}$ vanishes on the subspace $V_{h}$, those terms do not appear. The same procedure performed in $V_{h}$ and $\mathscr{V}_{h}$ yields a solution of (4.3), and since $\mathscr{L}$ is monotone, that solution is unique.

Finally we establish the convergence of the sequence of solutions of (4.2) to that of (4.3) as $\delta \rightarrow 0^{+}$. In the proof of existence above we showed that the sequence $\tilde{u}_{m}$ is bounded in $\mathscr{V}$ uniformly in $m$. Thus the limit $\tilde{u}$ is bounded, and from (4.8) we can see that the bound is independent of $\delta$. We will write $\tilde{u}_{\delta}$ to represent the solution of (4.2) and $\tilde{u}$ to represent the solution of (4.3). The set of solutions $\left\{u_{\delta}\right\}$ for $\delta \geq 0$ is bounded in $\mathscr{V}$ and in $L^{\infty}(0, T ; H)$, and $\mathscr{L} u_{\delta}$ is bounded in $\mathscr{V}^{\prime}$; thus there is a subsequence, also denoted by $\tilde{u}_{\delta}$, so that

$$
\begin{aligned}
& \tilde{u}_{\delta} \rightarrow \tilde{u}^{*} \text { weakly in } \mathscr{V}, \\
& \mathscr{L} \tilde{u}_{\delta} \rightarrow g \text { weakly in } \mathscr{V}^{\prime} \text { as } \delta \rightarrow 0^{+}, \text {and } \\
& \tilde{u}_{\delta}(T) \rightarrow \tilde{u}_{T}^{*} \text { in } H .
\end{aligned}
$$

Also, we have

$$
\delta\left(\tilde{u}_{\delta}^{\prime}, \tilde{u}_{\delta}\right)_{H}+\delta \mathscr{L} \tilde{u}_{\delta}\left(\tilde{u}_{\delta}\right)+\mathscr{M} \tilde{u}_{\delta}\left(\tilde{u}_{\delta}\right)=\delta \tilde{f}\left(\tilde{u}_{\delta}\right)
$$

Since $\mathscr{L} \tilde{u}_{\delta}$ is bounded in $\mathscr{V}^{\prime}, \tilde{u}_{\delta}$ is bounded in $\mathscr{V}$, and from (4.8) $\tilde{u}_{\delta}^{\prime}$ is bounded in $\mathscr{V}^{\prime}$, we may let $\delta \rightarrow 0$ and observe that $\mathscr{M}_{\delta}\left(\tilde{u}_{\delta}\right) \rightarrow 0$. Since $\mathscr{M}$ is linear and continuous, it is lower semicontinuous; thus $\mathscr{M} \tilde{u}^{*}\left(\tilde{u}^{*}\right) \leq \liminf \operatorname{in}_{\delta \rightarrow 0^{+}} \mathscr{M} \tilde{u}_{\delta}\left(\tilde{u}_{\delta}\right)=0$ and

$$
\int_{0}^{T} \int_{\Omega} \int_{\Gamma_{x}}\left|\gamma U^{*}-\lambda u^{*}\right|^{2} d s d x d t=0 \quad \text { where } \tilde{u}^{*}=\left[u^{*}, U^{*}\right] \in \mathscr{V} .
$$

In fact, the above shows that $\tilde{u}^{*} \in \mathscr{V}_{h}$. We will show that $\tilde{u}^{*}=\tilde{u}$; that is, the weak limit of $\left\{\tilde{u}_{\delta}\right\}$ is the unique solution of (4.3).

Choose $\varphi \in C^{1}[0, T]$ and $\tilde{v} \in V_{h}$. From (4.2) we obtain

$$
\int_{0}^{T}\left(\tilde{u}_{\delta}^{\prime}, \varphi \tilde{v}\right)_{H} d t+\int_{0}^{T} \mathscr{L} \tilde{u}_{\delta}(\varphi \tilde{v}) d t=\int_{0}^{T} \tilde{f}(\varphi \tilde{v}) d t
$$

(since $\varphi(t) \tilde{v} \in V_{h}$, the $\mathscr{M}$ term vanishes). Thus

$$
\begin{aligned}
& -\int_{0}^{T}\left(\tilde{u}_{\delta}, \varphi^{\prime} \tilde{v}\right)_{H} d t+\int_{0}^{T} \mathscr{L} \tilde{u}_{\delta}(\varphi \tilde{v}) d t \\
& \quad=\int_{0}^{T} \tilde{f}(\varphi \tilde{v}) d t+\left(\tilde{u}_{0}, \tilde{v}\right)_{H} \varphi(0)-\left(\tilde{u}_{\delta}(T), \tilde{v}\right)_{H} \varphi(T) .
\end{aligned}
$$


Letting $\delta \rightarrow 0^{+}$in the above gives

$$
-\int_{0}^{T}\left(\tilde{u}^{*}, \varphi^{\prime} \tilde{v}\right)_{H} d t+\int_{0}^{T} g(\varphi \tilde{v}) d t=\int_{0}^{T} \tilde{f}(\varphi \tilde{v}) d t+\left(\tilde{u}_{0}, \tilde{v}\right)_{H} \varphi(0)-\left(\tilde{u}_{T}^{*}, \tilde{v}\right) \varphi(T) ;
$$

thus,

$$
\int_{0}^{T}\left(\tilde{u}^{* \prime}, \varphi \tilde{v}\right)_{H} d t+\int_{0}^{T} g(\varphi \tilde{v}) d t=\int_{0}^{T} \tilde{f}(\varphi \tilde{v}) d t+\left(\tilde{u}^{*}(T)-\tilde{u}_{T}^{*}, \tilde{v}\right) \varphi(T) .
$$

Since this holds for all $\tilde{v} \in \mathscr{V}_{h}$ and $\varphi \in C^{1}[0, T]$, we have

$$
\tilde{u}^{* \prime}+g=\tilde{f} \text { in } \mathscr{V}_{h}^{\prime}, \quad \tilde{u}^{*}(T)=\tilde{u}_{T}^{*},
$$

and $\tilde{u}_{\delta}^{\prime} \rightarrow \tilde{u}^{* \prime}$ weakly in $\mathscr{V}_{h}^{\prime}$.

In (4.2) let $\tilde{\varphi}=\tilde{u}_{\delta}-\tilde{u}^{*}$. Then

$$
\begin{aligned}
\int_{0}^{T} \mathscr{L} \tilde{u}_{\delta}\left(\tilde{u}_{\delta}-\tilde{u}^{*}\right) d s= & \int_{0}^{T} \tilde{f}\left(\tilde{u}_{\delta}-\tilde{u}^{*}\right) d s-\int_{0}^{T}\left(\tilde{u}_{\delta}^{\prime}, \tilde{u}_{\delta}-\tilde{u}^{*}\right)_{H} d s \\
& +\frac{1}{\delta}\left(\mathscr{M} \tilde{u}^{*}-\mathscr{M} \tilde{u}_{\delta}\right)\left(\tilde{u}_{\delta}-\tilde{u}^{*}\right) .
\end{aligned}
$$

is monotone; so by taking limsup as $\delta \rightarrow 0^{+}$we obtain

$$
\begin{aligned}
\underset{\delta \rightarrow 0}{\limsup } \int_{0}^{T} \mathscr{L} \tilde{u}_{\delta}\left(\tilde{u}_{\delta}-\tilde{u}^{*}\right) d s & \leq \limsup _{\delta \rightarrow 0} \int_{0}^{T}\left(\tilde{u}_{\delta}^{\prime}, \tilde{u}^{*}\right)_{H} d s-\liminf _{\delta \rightarrow 0^{+}} \int_{0}^{T}\left(\tilde{u}_{\delta}^{\prime}, \tilde{u}_{\delta}\right)_{H} d s \\
& =\int_{0}^{T}\left(\tilde{u}^{* \prime}, \tilde{u}^{*}\right)_{H} d s-\liminf _{\delta \rightarrow 0^{+}} \frac{1}{2}\left\|\tilde{u}_{\delta}(T)\right\|_{H}^{2}+\frac{1}{2}\left\|\tilde{u}_{0}\right\|_{H}^{2} \\
& =\frac{1}{2}\left\|\tilde{u}^{*}(T)\right\|_{H}^{2}-\liminf _{\delta \rightarrow 0^{+}} \frac{1}{2}\left\|\tilde{u}_{\delta}(T)\right\|_{H}^{2} .
\end{aligned}
$$

Since the $H$-norm is weakly lower semicontinuous and $\tilde{u}_{\delta}(T)-u^{*}(T)$ in $H$,

$$
\frac{1}{2}\left\|\tilde{u}^{*}(T)\right\|_{H}^{2} \leq \liminf _{\delta \rightarrow 0^{+}} \frac{1}{2}\left\|\tilde{u}_{\delta}(T)\right\|_{H}^{2} .
$$

Thus

$$
\limsup _{\delta \rightarrow 0} \int_{0}^{T} \mathscr{L} \tilde{u}_{\delta}\left(\tilde{u}_{\delta}\right) d s \leq \int_{0}^{T} g\left(\tilde{u}^{*}\right) d s .
$$

Since $\mathscr{L}$ is type- $M$ in $\mathscr{V}, \tilde{u}_{\delta} \rightarrow \tilde{u}^{*}$, and $\mathscr{L} u_{\delta} \rightarrow g$, this shows that

$$
\mathscr{L} \tilde{u}^{*}=g \text { in } \mathscr{V}_{h}^{\prime}
$$

so $\tilde{u}^{*}$ is a solution of $(4.3)$ and $\tilde{u}^{*}=\tilde{u}$ by uniqueness.

5. Remarks. We have shown that $(3.5)$ and $(3.5)^{\prime}$ have unique solutions and that the two models which they represent are related. We remarked previously that allowing $\delta \rightarrow 0^{+}$formally transformed the regularized model into the matched model. We have substantiated that observation by showing that the solutions $u_{\delta}$ converge to the solution of the matched model.

Note also that the variational form (in $(3.2)^{\prime}$ for example) leads directly back to (3.1 $)^{\prime}$. This is confirmation that our choice of $Q$, the exchange term in the physical model, is the correct one. 
Finally note that the models and results here could be generalized or extended in several ways. For example, the assumption of a layered structure could be dropped and the secondary flux computed in all three coordinate directions. This provides a model for partially fissured media in which the cells are not isolated from each other and the secondary flux results from direct cell-to-cell diffusion driven by the pressure gradient in the fissure system. In (3.1c) we might choose $\mu$ to be something other than a constant. If, for example, $\mu$ is assumed to be a monotone graph that is also a subgradient operator, an approach similar to that in [17] might be used to show existence of a solution. As stated earlier, Dirichlet boundary conditions on $\partial \Omega$ are not necessary; so some generalization is also possible in that respect. Finally, if additional assumptions about the differentiability of $A$ and $B$ and the smoothness of $\Gamma_{x}$ and $\partial \Omega$ were made, then it might be possible to say more about the regularity of $u$ and $U$.

\section{BIBLIOGRAPHY}

[1] R. A. Adams, Sobolev Spaces, Academic Press, New York, 1975

[2] T. Arbogast, Analysis of the simulation of single phase flow through a naturally fractured reservoir, SIAM J. Numer. Anal. 26, 12-29 (1989)

[3] T. Arbogast, The double porosity model for single phase flow in naturally fractured reservoirs, Inst. Math. Appl. 295 (1987)

[3] T. Arbogast, J. Douglas, and U. Hornung, Modeling of naturally fractured petroleum reservoirs by formal homogenization techniques, in Frontiers in Pure and Applied Mathematics (R. Dautray, ed.), Elsevier, Amsterdam, 1991, pp. 1-19

[5] G. I. Barenblatt, I. P. Zheltov, and I. N. Kochina, Basic concepts in the theory of seepage of homogeneous liquids in fissured rocks, J. Appl. Math. Mech. 24, 1286-1303 (1960)

[6] N. S. Boulton and T. D. Streltsova-Adams, Unsteady flow to a pumped well in an unconfined fissured aquifer, Advances in Hydroscience, 1978, pp. 357-423

[7] E. A. Coddington and N. Levinson, Theory of Ordinary Differential Equations, McGraw-Hill Book Company, New York, 1955

[8] U. Hornung and R. E. Showalter, Diffusion models for fractured media, J. Math. Anal. Appl. 147, 69-80 (1990)

[9] E. Kasap and L. W. Lake, Calculating the effective permeability tensor of a gridblock, Society of Petroleum Engineers Formation Evaluation, June 1990, pp. 192-200

[10] J. L. Lions, Quelques Méthodes de Résolutions des Problèmes aux Limites Non Linéaires, Dunod, Paris, 1969

[11] R. K. Miller, An integrodifferential equation for rigid heat conductors with memory, Jour. Math. Anal. Appl. 66, 313-332 (1978)

[12] J. W. Nunziato, On heat conduction in materials with memory, Quart. Appl. Math. 29, 187-204 (1971)

[13] W. Rudin, Real and Complex Analysis, McGraw-Hill Book Company, New York, 1987

[14] R. E. Showalter, Hilbert Space Method for Partial Differential Equations, Pitman, 1977

[15] R. E. Showalter, Monotone Operators in Banach Space and Nonlinear Partial Differential Equations (to appear)

[16] R. E. Showalter and N. J. Walkington, Micro-structure models of diffusion in fissured media, Jour. Math. Anal. Appl. 155, 1-20 (1991)

[17] R. E. Showalter and N. J. Walkington, Diffusion of fluid in a fissured medium with micro-structure, SIAM Jour. Math. Anal. 22, 1702-1722 (1991) 